\title{
Staged bilateral single-port thoracoscopic lung volume reduction surgery: A report of 11 cases
}

\author{
MIAO ZHANG, HENG WANG, XUE-FENG PAN, WEN-BIN WU and HUI ZHANG
}

Department of Thoracic Surgery, Xuzhou Central Hospital

Affiliated to Southeast University, Xuzhou, Jiangsu 221009, P.R. China

Received January 29, 2016; Accepted August 18, 2016

DOI: $10.3892 /$ etm.2016.3702

\begin{abstract}
The aim of the present study was to investigate the feasibility and efficacy of staged bilateral single-port thoracoscopic lung volume reduction surgery (LVRS) for patients with chronic obstructive pulmonary emphysema (COPE). Eleven male patients with a mean age of $60.27 \pm 12.11$ years with bilateral COPE and bullae were admitted to the Department of Thoracic Surgery, Xuzhou Central Hospital from January 2013 to June 2014. The patients underwent staged bilateral single-port thoracoscopic LVRS. The hyperinflated bullae were resected using endoscopic staplers (Endo-GIA), followed by continuous suture and biological glue for reinforcement of the margin. In addition, pulmonary function, blood gas assay, 6-min walk distance (6MWD) and life quality evaluated by a short form 36-item health survey questionnaire (SF-36) were recorded before and after LVRS, respectively. All the patients survived after surgery. The chest tube drainage time was $9.09 \pm 1.31$ days and postoperative hospital stay was $15.73 \pm 2.75$ days, with 5 cases of persistent air leakage and 7 cases of pulmonary infection which were finally cured. The patients were followed up for 3 to 12 months, and the pulmonary function, partial pressure of oxygen $\left(\mathrm{pO}_{2}\right), 6 \mathrm{MWD}$ and life quality after unilateral or bilateral LVRS were improved compared to these parameters before surgery. However, there was no significant difference between unilateral and bilateral LVRS in terms of life quality. In conclusion, staged bilateral single-port thoracoscopic LVRS may improve the short-term life quality of patients with COPE.
\end{abstract}

\section{Introduction}

Chronic obstructive pulmonary emphysema (COPE) behaves as progressive dyspnea, which seriously attenuates the quality of life of patients. The study of the National Emphysema Treatment Trial (NETT) has indicated that lung function

Correspondence to: Dr Hui Zhang, Department of Thoracic Surgery, Xuzhou Central Hospital Affiliated to Southeast University, Xuzhou, Jiangsu 221009, P.R. China

E-mail: wpvlyqo63532@163.com

Key words: chronic obstructive pulmonary emphysema, single-port thoracoscopy, lung volume reduction surgery, COPE, LVRS and the quality of life of patients after lung volume reduction surgery (LVRS) were superior to medical treatment (1). Studies have shown that the improved duration course of staged bilateral LVRS was superior to unilateral or simultaneous bilateral LVRS (2). We examined a total of 11 patients with bilateral COPE combined with bullae from January 2013 to June 2014. The patients underwent staged bilateral single-port thoracoscopic LVRS treatment, which significantly improved their lung function and quality of life.

\section{Patients and methods}

Patients. The selection of single-port thoracoscopic LVRS patients was carried out as defined in NETT (3). Eleven male patients with bilateral COPE and bullae who underwent staged bilateral single-port thoracoscopic LVRS in our hospital from January 2013 to June 2014 were enrolled in our study. Mean patient age was $60.27 \pm 12.11$ years and body weight index was $34.35 \pm 0.69$. Among the total cases, 1 case was confirmed with inhomogeneous emphysema and bullae combined with bronchial dilatation under CT imaging before surgery. Two cases were confirmed with a long-term history of chest distress and asthmatic suffocation. These patients had a medicine treatment course of $10.09 \pm 4.25$ years, combined with pneumothorax upon admission, with relatively poor recruitment maneuver of lung after thoracic close drainage. Before surgery, the patient was required to stop smoking and was treated by atomization, coughing and expectorating to effectively control asthmatic suffocation, bronchial dilatation and pulmonary infection. In addition, the patient also received respiratory function exercise and standardized medication to prevent being exposed to allergen. After the lung function was confirmed to be consistent with the operation indications, single-port thoracoscopic LVRS was performed under general intravenous anesthesia. Parameters of the lung function and arterial blood gas one week before surgery included: forced expiratory volume in the first second (FEV1), 1.37 \pm 0.24 liters; arterial oxygen partial pressure $\left(\mathrm{pO}_{2}\right), 66.91 \pm 2.95 \mathrm{mmHg}$; carbon dioxide partial pressure $\left(\mathrm{pCO}_{2}\right), 46.82 \pm 2.96 \mathrm{mmHg}$ and 6-minute walk distance (6MWD), 297.73 $\pm 8.91 \mathrm{~m}$ (Table I).

\section{Methods}

Surgical method. Double lumen endotracheal intubation was performed, with atracurium, fentanyl and propofol used for 
Table I. Pulmonary function and 6MWD in patients before and after LVRS.

\begin{tabular}{lccccc}
\hline & FEV1 (liters) & FEV1 $(\%)$ & $\mathrm{PO}_{2}(\mathrm{mmHg})$ & $\mathrm{pCO}_{2}(\mathrm{mmHg})$ & 6MWD (m) \\
\hline Pre-surgery & $1.37 \pm 0.24$ & $52.41 \pm 3.60$ & $66.91 \pm 2.95$ & $46.82 \pm 2.96$ & $297.73 \pm 8.91$ \\
3 months after unilateral surgery & $1.69 \pm 0.20$ & $60.43 \pm 3.35$ & $74.36 \pm 5.64$ & $42.73 \pm 2.83$ & $433.36 \pm 19.10$ \\
3 months after bilateral surgery & $1.75 \pm 0.19$ & $65.60 \pm 4.77$ & $79.09 \pm 3.78$ & $40.27 \pm 2.05$ & $472.09 \pm 33.08$ \\
\hline
\end{tabular}

6MWD, 6-min walk distance; LVRS, lung volume reduction surgery. Data are expressed as mean \pm SD.
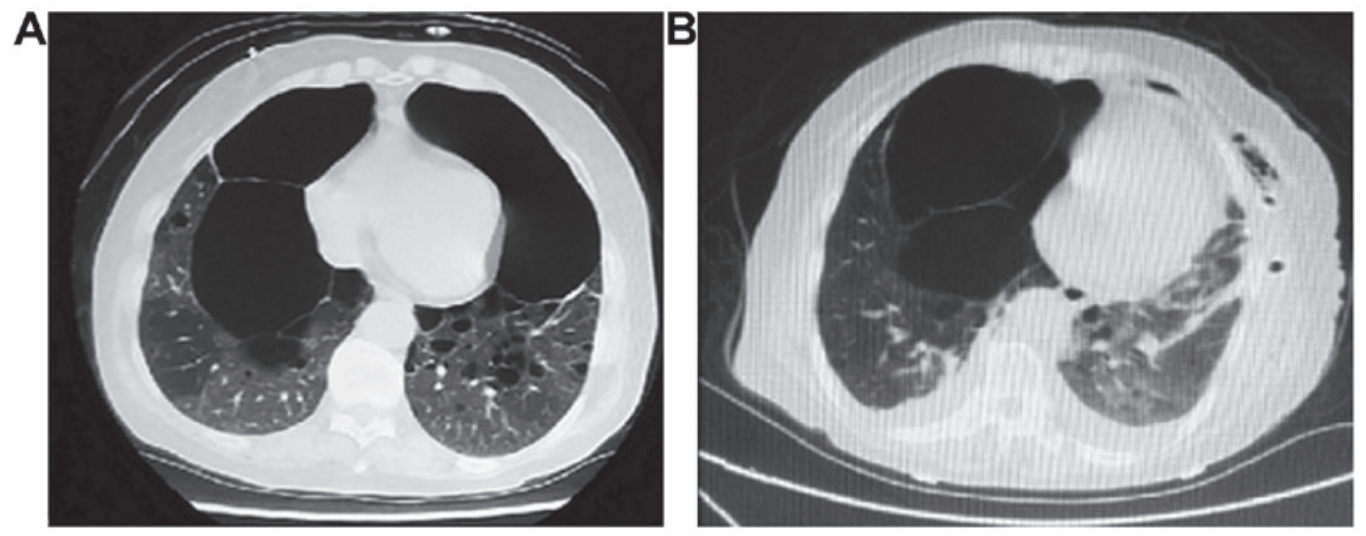

Figure 1. Computed tomography (CT) of patients with COPE combined with giant bullae. (A) Before surgery, (B) one week after unilateral LVRS surgery. COPE, chronic obstructive pulmonary emphysema; LVRS, lung volume reduction surgery.

general anesthesia. Subsequently, unilateral lung ventilation was carried out; holes were made in the fifth or sixth rib intercostal space of the anterior axillary line and the length of the incision was $4.54 \pm 0.47 \mathrm{~cm}$. The target region was determined according to CT and the condition was observed by thoracoscope. The endoscopic cutting device (Endo-GIA) was used to excise the overinflated lung tissues in the target region. The cutting edges were overlapped and consecutively the incisal margins were sutured by 4-0 prolene followed by biological protein glue. The inferior pulmonary ligament was loosened and the parietal pleura was rubbed and pleural perfusion by group A Streptococcus (OK-432) was performed to promote pleural adhesion. After surgery, the lung was dilated to exclude the incisal margin from air leakage. A no. 7 chest tube was placed between the second intercostal space in the midclavicular line and one no. 26 chest tube between the eighth and ninth rib. After surgery, $1 \mu \mathrm{g} / \mathrm{ml}$ fentanyl automatic control vein pump was used to alleviate pain for $48 \mathrm{~h}$. The patients were sent to ICU for continuous monitoring and treatment for $1.73 \pm 0.64$ days. The patients were assisted with independent expectoration, strengthening of respiratory tract nursing, and the time of tracheal intubation was shortened. Three to four months after unilateral LVRS when the lung function and general condition of the patients were improved, bilateral LVRS was carried out.

Evaluation of the quality of life. Lung function and arterial blood gas were determined under non-oxygen uptake state and 6MWD examinations one week before surgery, three months after unilateral LVRS and three months after bilateral LVRS. In the meanwhile, the patients were guided to complete the short form 36-item health survey (SF-36) and their scores was analyzed for evaluation of their quality of life.

Statistical analysis. SPSS 19.0 software (SPSS Inc., Chicago, IL, USA) was applied for statistical analysis. Continuous variables are presented as mean \pm SD. The Student's t-test or Mann-Whitney U test was applied for comparisons between groups. The $\chi^{2}$ or Fisher's exact test was used for comparisons between groups with regard to categorical variables and enumeration data. $\mathrm{P}<0.05$ was considered to indicate a statistically significant difference.

\section{Results}

None of the patients included in our study had perioperative death. Surgery lasted for $126.36 \pm 14.40 \mathrm{~min}$, post-surgery mechanical ventilation lasted for $9.68 \pm 1.49 \mathrm{~h}$, post-surgery thoracic drainage tube lasted for $9.09 \pm 1.31$ days, and hospitalization lasted for $15.73 \pm 2.75$ days. Among the cases, 5 cases had lung air leakage for $10.40 \pm 2.97$ days, 7 cases had pulmonary infection, and 5 cases had atelectasis. All patients were cured and there was no other complications such as heart and lung failure or thrombosis. One case was relatively typical, and was diagnosed as COPE with giant bullae (also known as vanishing lung syndrome). After unilateral LVRS surgery, parameters of lung function of the patients were improved significantly compared with those prior to surgery (Fig. 1).

The pulmonary function, arterial blood gas analysis and activity of the daily living of the patients before surgery were compared three months after unilateral LVRS and three months after bilateral surgery. Comparative results 


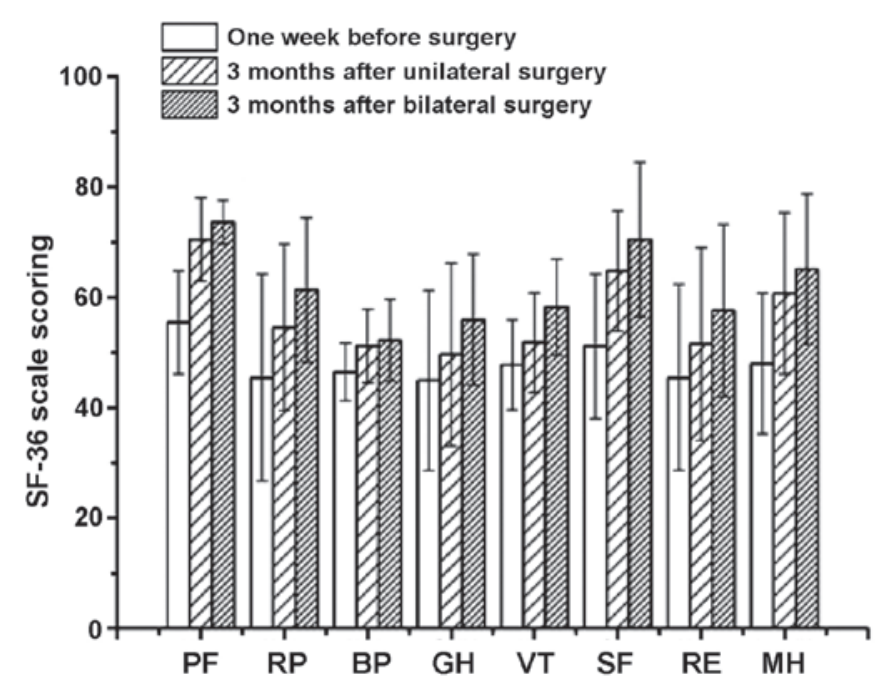

Figure 2. Life quality score of patients before and after LVRS surgery. LVRS, lung volume reduction surgery. PF, physical functioning; RP, role-physical BP, bodily pain; GH, general health; VT, vitality; SF, social functioning; RE. role-emotional; $\mathrm{MH}$, mental health.

showed that the proportion of FEV1 in predicting value [FEV1 (\%)], arterial $\mathrm{PO}_{2}$ and 6MWD were increased compared with these parameters before surgery while arterial $\mathrm{pCO}_{2}$ was reduced compared with that before surgery; there was no significant difference in the pulmonary function and blood gas analysis of the patients between unilateral and bilateral LVRS ( $\mathrm{p}>0.05$ ) (Table I).

Moreover, the SF-36 included items such as physical functioning (PF), role-physical (RP), bodily pain (BP), general health $(\mathrm{GH})$, vitality (VT), social functioning (SF), role-emotional $(\mathrm{RE})$ and mental health $(\mathrm{MH})$ and were used to evaluate preliminarily the quality of life. Fig. 2 shows the scores for the SF-36 scale. Compared with pre-surgery, short-term life quality of patients after LVRS surgery was greatly improved and patients could take care of themselves. Comparison of bilateral and unilateral LVRS surgery was not statistically significant in terms of lung function and quality of life $(\mathrm{p}>0.05)$.

\section{Discussion}

Physical and psychology effects of thoracic surgery on patients are relatively slight and small. Patients gain physiologic functions very soon after thoracic surgery (4). The indications for single-port thoracoscope have been gradually extended (5). Gonzalez-Rivas et al reported on the single-port thoracoscope bronchus and pulmonary artery double sleeve pulmonary lobectomy (6). The mechanism of LVRS involves the excision of $20-40 \%$ of the overventilated lung tissues to increase their elasticity, adjustment of the ventilation/blood flow ratio, reduction of the pulmonary heterogeneity to alleviate the respiratory pump failure, and improvement of alveolar gas exchange. Additionally, the improvement of pulmonary function and quality of life of the patients after LVRS could last for 3-4 years (7). For COPE dominated by the superior lobe, we recommend LVRS (8). It was reported that the lung function and quality of life of COPE patients was significantly improved 6 months after simultaneous bilateral thoracoscopic LVRS (9). This may be because ventilation efficiency was greatly improved after LVRS surgery (10).

The incision of single-port thoracoscope was between the fifth and sixth rib between the anterior axillary line and the midaxillary line. An endoscopic cut stapler of $5 \mathrm{~mm}$ was selected, with $30^{\circ}$ angle and rotatable head (11). After one-lung ventilation, due to obstructive airway obstruction, patients in this group sometimes had insufficient lung collapse and extensive pleural adhesion, thus greatly limiting the vision and operation. To avoid the residue of pulmonary bulla tissues and protect the normal lung tissues, the incision had to be performed several times, thus resulting in extended surgical time and a waste of medical resources. The single direction incison procedure by Liu and the preoperative CT reconstruction may be utilized to precisely locate the target lung tissues (12), thus it was a favorable method to overcome such a limit and save medical resources. Major complications of LVRS included pulmonary atelectasis, pulmonary infection and persistent pulmonary leakage. Overlap of the incisal margins and interlocking suture by 4-0 prolene thread, combined with biogel significantly reduced post-surgery air leakage (13). The chest was closed after $10 \mathrm{~min}$ of lung filling. Conventionally, 2 chest tubes were inserted individually, with the ends of the tube placed into the top and bottom of the thoracic cavity. Special care was given to avoid that the tube did not touch the heart so as to prevent arrhythmia such as atrial fibrillation after surgery. The patient were provided with nutrition, assistance in coughing and expectorating and off-bed activity and were regularly monitored by chest X-ray to clarify the degree of pulmonary re-expansion. It has been reported that OK-432 effectively reduces the recurrence of pneumothorax and the adverse effects were relatively minimal (14). We used OK-432 to perform thoracic cavity perfusion on patients after pneumothorax and pulmonary trachea operation to promote pleural adhesion and have received quite favorable curative effects.

The major causes of post-surgery pulmonary infection in patients with chronic obstructive pulmonary disease is increased sputum secretion and obstructed discharge, most of which are complicated by tracheal colonization bacteria. The airway passage needs to be healthy before surgery and thus sensitive antibiotics should be selected before and after surgery (15). Furthermore, the lung function of patients with chronic obstructive lung disease is relatively poor, thus assistant sputum excretion and rehabilitation therapy after surgery was required (16). In the present study, patients were treated with conventional small dose hormone aerosol inhalation to control airway inflammation.

By contrast, patients in our study received partial resection of the pulmonary lobe, including pulmonary bullae so as to preserve the reserve function of their lung. Beckers et al reported that, compared with pulmonary lobe partial resection, lobectomy could better improve the curative effects on the lung function of patients with severe emphysema (17). A meta-analysis by Iftikhar et al indicated that bronchoscopic lung volume reduction (BLVR) was a simple replacement therapy of emphysema (18). Chen et al confirmed the curative effects of lung transplantation and LVRS on treating end-stage emphysema, indicating that compared with LVRS, lung transplantation could better improve lung function of patients, 
blood gas analysis and quality of life after surgery. Therefore, it was suggested that if conditions permitted, lung transplantation be considered the first choice of surgical treatment for patients with end-stage emphysema (19). However, Trotter and Hopkins demonstrated the role of lung volume reduction and lung transplantation for treating emphysema. Since there were few lung donors available, endobronchial valve therapy, bronchial vapor ablation and LVRS should be considered (20). Shah and Herth reported that endobronchial valve therapy could significantly improve the lung function and activity endurance of patients with emphysema (21).

In conclusion, staged bilateral single-port thoracoscopic LVRS is safe and effective for treating patients with COPE combined with pulmonary bullae. Since the sample number of our study was relatively small, its short-term curative effect still requires a multi-center, large scale study and its long-term curative effect requires closer follow-up.

\section{References}

1. Kaplan RM, Sun Q, Naunheim KS and Ries AL: Long-term follow-up of high-risk patients in the National Emphysema Treatment Trial. Ann Thorac Surg 98: 1782-1789, 2014.

2. Oey IF, Morgan MD, Spyt TJ and Waller DA: Staged bilateral lung volume reduction surgery-the benefits of a patient-led strategy. Eur J Cardiothorac Surg 37: 846-852, 2010.

3. Ginsburg ME, Thomashow BM, Yip CK, DiMango AM, Maxfield RA, Bartels MN, Jellen P, Bulman WA, Lederer D, Brogan FL, et al: Lung volume reduction surgery using the NETT selection criteria. Ann Thorac Surg 91: 1556-1560, 2011.

4. Zhou Y, Liu L, Yu, P, Su, J, Shen C, Pu Q, Ma L and Che G: Fast-track recovery of cardiopulmonary function after complete video-assisted thoracoscopic lobectomy. Chin J Clin Thorac Cardiovasc Surg 20: 168-171, 2013.

5. Ng CS, Rocco G, Wong RH, Lau RW, Yu SC and Yim AP: Uniportal and single-incision video-assisted thoracic surgery: the state of the art. Interact Cardiovasc Thorac Surg 19: 661-666, 2014.

6. Gonzalez-Rivas D, Delgado M, Fieira E and Fernandez R: Double sleeve uniportal video-assisted thoracoscopic lobectomy for non-small cell lung cancer. Ann Cardiothorac Surg 3: E2, 2014.

7. Wei H, Bo D, Wang R, et al: Effect of lung volume reduction surgery for patients with emphysema. China J Thorac Cardiovasc Surg 28: 312-314, 2012.
8. Clark SJ, Zoumot Z, Bamsey O, Polkey MI, Dusmet M, Lim E, Jordan S and Hopkinson NS: Surgical approaches for lung volume reduction in emphysema. Clin Med Lond 14: 122-127, 2014.

9. Wei Q, Zhang A, Lin Z et al: Bilateral lung volume reduction by video assisted thoracic surgery for 21 cases. Chin J Clin Thorac Cardiovasc Surg 19: 444-446, 2012.

10. Armstrong HF, Dussault NE, Thirapatarapong W, Lemieux RS, Thomashow BM and Bartels MN: Ventilatory efficiency before and after lung volume reduction surgery. Respir Care 60: 63-71, 2015.

11. Che G and Liu L: Advancement and progress of single-port video-assisted thoracoscopic surgery in the treatment of thoracic diseases. Chin J Clin Thorac Cardiovasc Surg 19: 181-184, 2012.

12. Liu C and Liu L: Uniportal VATS: a sublimation of micro-invasive lung cancer resection. Zhongguo Fei Ai Za Zhi 17: 527-530, 2014 (In Chinese).

13. Rathinam S, Naidu BV, Nanjaiah P, Loubani M, Kalkat MS and Rajesh PB: BioGlue and Peri-strips in lung volume reduction surgery: pilot randomised controlled trial. J Cardiothorac Surg 4: $37,2009$.

14. How CH, Tsai TM, Kuo SW, Huang PM, Hsu HH, Lee JM, Chen JS and Lai HS: Chemical pleurodesis for prolonged postoperative air leak in primary spontaneous pneumothorax. J Formos Med Assoc 113: 284-290, 2014.

15. Zhi X: Expert consensus of airway management in Department of Thoracic Surgery. Chin J Clin Thorac Cardiovasc Surg 20: 251-255, 2013.

16. Mei J, Liu L, Tang M, Xu N, Pu Q, Liu C, Ma L, Shi H and Che G: Airway bacterial colonization in patients with non-small cell lung cancer and the alterations during the perioperative period. J Thorac Dis 6: 1200-1208, 2014.

17. Beckers F, Lange N, Koryllos A, Picchioni F, Windisch W and Stoelben E: Unilateral lobe resection by video-assisted thoracoscopy leads to the most optimal functional improvement in severe emphysema. Thorac Cardiovasc Surg: Dec 23, 2014 (Epub ahead of print)

18. Iftikhar IH, McGuire FR and Musani AI: Efficacy of bronchoscopic lung volume reduction: a meta-analysis. Int J Chron Obstruct Pulmon Dis 9: 481-491, 2014.

19. Chen Y, Chen J and Wang Z: analysis of clinical outcomes of lung volume reduction surgery and lung transplantation on end-stage emphysema. Chin J Clin Thorac Cardiovasc Surg 19: 141-144, 2012.

20. Trotter MA and Hopkins PM: Advanced therapies for COPD-What's on the horizon? Progress in lung volume reduction and lung transplantation. J Thorac Dis 6: 1640-1653, 2014.

21. Shah PL and Herth FJ: Current status of bronchoscopic lung volume reduction with endobronchial valves. Thorax 69: 280-286, 2014. 